Cahiers $d u$ MONDE RUSSE

\section{Cahiers du monde russe}

Russie - Empire russe - Union soviétique et États indépendants

$50 / 2-3 \mid 2009$

L'Europe orientale, 1650-1730. Crises, conflits et renouveau

\title{
Zapisnye votčinnye knigi Pomestnogo prikaza 1626-1657 gg.A.V. ANTONOV, A. BERELOWITCH, V.D. NAZAROV, eds.
}

, [Registres de transactions patrimoniales de l'office des fiefs, 1626-1657], Moscou : «Drevnehranilišče », 2010, 1658 p.

\section{André Berelowitch}

\section{(2) OpenEdition}

\section{Journals}

\section{Édition électronique}

URL : https://journals.openedition.org/monderusse/9746

DOI : 10.4000/monderusse. 9746

ISSN : 1777-5388

\section{Éditeur}

Éditions de l'EHESS

\section{Édition imprimée}

Date de publication : 15 septembre 2009

ISBN : 978-2-7132-2260-3

ISSN : $1252-6576$

\section{Référence électronique}

André Berelowitch, «Zapisnye votčinnye knigi Pomestnogo prikaza 1626-1657 gg.A.V. ANTONOV, A. BERELOWITCH, V.D. NAZAROV, eds. », Cahiers du monde russe [En ligne], 50/2-3 | 2009, mis en ligne le 14 janvier 2013, consulté le 03 septembre 2022. URL : http://journals.openedition.org/monderusse/ 9746 ; DOI : https://doi.org/10.4000/monderusse.9746

Ce document a été généré automatiquement le 3 septembre 2022.

Tous droits réservés 


\section{Zapisnye votčinnye knigi Pomestnogo prikaza 1626-1657 gg.A.V. ANTONOV, A. BERELOWITCH, V.D. NAZAROV, eds.}

, [Registres de transactions patrimoniales de l'office des fiefs, 1626-1657], Moscou : «Drevnehranilišče », 2010, 1658 p.

André Berelowitch

\section{A.V. ANTONOV, A. BERELOWITCH, V.D. NAZAROV, eds., Zapisnye votčinnye knigi Pomestnogo prikaza 1626-1657 gg., [Registres de transactions patrimoniales de l'Office des fiefs, 1626-1657], Moscou : « Drevnehranilišče », 2010, 1658 p.}

$1 \mathrm{Si}$, contrairement à l'usage, je présente un ouvrage que j'ai contribué à rédiger, c'est que ma contribution est minime. Le mérite d'avoir réalisé ce travail monumental (26.5 par 18 par $9 \mathrm{~cm}$ ) revient essentiellement à Anton Vladislavovič Antonov, responsable au RGADA des archives foncières de la monarchie moscovite (fonds 1209) et grand connaisseur de ce type de documents ${ }^{1}$. C'est lui qui a conçu la structure du volume et les critères d'analyse des documents, lui encore qui a dépouillé la plus grande partie des livres. Le regretté Viktor Jur'evič Belikov, décédé en 2005, avait eu le temps de dépouiller 10 registres (sur un total de 46), et Elisabeth Teiro a analysé le registre $n^{\circ}$ 5987. L'entreprise enfin n'aurait pu aboutir sans le soutien financier que la Fondation de la Maison des sciences de l'homme a généreusement accordé, de 2003 à 2006, au projet n 659, « Notariat d'État en Russie au XVII siècle ».

2 À partir du milieu du xVI siècle, semble-t-il, les transactions foncières, celles avant tout qui transféraient d'un lignage à un autre la possession d'un bien patrimonial (une votčina, à distinguer du pomest'e, ou "fief», " bénéfice", accordé en précaire par le tsar à ses serviteurs), devaient obligatoirement être enregistrées dans les bureaux de la monarchie. 
Cette obligation est mentionnée dans divers documents des années 1558-1562, mais le terme technique zapisnaja votčinnaja kniga n'apparaît qu'un peu plus tard, dans un document de 1606. Aucun registre antérieur à 1626 n'a été conservé, bien que certains d'entre eux, ceux de 1574/1575 et de 1587/1588 par exemple, soient expressément mentionnés dans une supplique de l'abbé de Saint-Joseph-de-Volokolamsk ("Introduction» de Vladislav Nazarov, p. 12-15). Les 46 registres de 1626-1657, qui totalisent près de 40000 folios, sont apparemment moins complets (à moins que les transactions n'aient été moins nombreuses) que les 150 registres, encore inexplorés, qui couvrent la période 1648-1748 («Introduction » d'Anton Antonov, p. 3).

3 La procédure, telle qu'elle apparaît dans les documents de la première moitié du XVII siècle, est déjà bien rodée: qu'il s'agisse d'une vente, de l'aliénation au profit du créancier d'un domaine mis en gage par un débiteur insolvable, ou d'un transfert sans contrepartie monétaire (héritage, dot, échange), les parties doivent comparaître, ou se faire représenter par un parent ou un fondé de pouvoir, devant les responsables de l'office des fiefs (Pomestnyj prikaz). Elles exposent le contenu de la transaction et fournissent à l'appui diverses pièces qui sont recopiées « mot pour mot » par les scribes de l'office et incluses dans le procès-verbal. Dans le cas le plus simple, il s'agit d'un acte de vente ou d'un contrat de prêt hypothécaire. Mais lorsqu'il devient nécessaire de prouver ses droits sur la terre qui fait l'objet de la transaction, l'héritier devra produire un testament, le gendre un contrat de mariage, l'office peut demander aussi des extraits de cadastres ou des contrats de vente antérieurs, etc. Ce sont les procès-verbaux intégraux de chaque affaire qui figurent dans les registres de transactions patrimoniales. Ils peuvent aller de quelques folios (quand la transaction est simple et qu'il n'y a pas contestation) à des centaines de pages, par exemple lorsque différents lignages se disputent un même bien : dans ce dernier cas, l'office des fiefs, étant investi, comme les autres départements ministériels (« secrétariats »), de la fonction judiciaire, instruit et arbitre un véritable procès.

4 La majeure partie de l'ouvrage (p. 17-1336) est consacrée à la description des registres : le dossier (delo) ouvert pour chaque transaction est analysé pièce par pièce. Une liste chronologique des transactions (p. 1338-1472), un index des noms (p. 1473-1588) et des lieux (p. 1589-1656) récapitulent les données à l'usage des chercheurs. On peut regretter (mais le volume est déjà énorme) l'absence d'un index terminologique.

5 Un certain nombre de particularités, comme j'ai essayé de le montrer («Introduction», p. 5-6), distinguent ces procès-verbaux moscovites des sources notariales françaises. Du point de vue de l'histoire sociale, toutefois, ce sont les ressemblances qui comptent, et à cet égard il n'est pas exagéré de considérer la parution du volume comme un véritable événement.

6 Les sources dont disposait jusqu'à présent l'historien de cette période paperassière sont abondantes, il est vrai, mais proviennent dans une large mesure d'organismes administratifs : la correspondance privée est rare, les lignages dont les archives sont parvenues jusqu'à nous - l'exception. Il est difficile dans ces conditions de se faire une idée tant soit peu précise de la vie des familles. Nous ignorons souvent leur généalogie (les documents officiels sont incomplets, et ne s'intéressent qu'aux mâles), leurs alliances, leur patrimoine immobilier que les cadastres ne décrivent que de temps à autre, et toujours imparfaitement. Sur tous ces points, les registres de transactions patrimoniales apportent des informations précieuses et probablement uniques, dont on peut augurer qu'à terme elles bouleverseront la discipline. 
7 Non seulement les domaines sont décrits, le plus souvent de façon sommaire, parfois amoureusement et en détail, par le vendeur, mais nous assistons en outre, au fil des pages, à la constitution d'une fortune foncière par une famille en pleine ascension sociale, à des achats systématiques de petits domaines ouvrant la voie au latifundium, ou au contraire à la dilapidation d'un capital immobilier par des ventes répétées à brefs intervalles. Autrement dit, nous disposons d'une source sérielle qui nous livre la dynamique de la propriété noble, ses modes de transmission et son évolution sur une période de temps allant jusqu'à trois générations.

8 Les testaments, et parfois la procédure administrative elle-même, nous livrent les filiations détaillées, sans omettre les femmes, les noms et les familles d'origine des conjoints successifs, les dates de décès des ayants droit, les legs des oncles et tantes à leurs neveux et nièces, les fondations pieuses et les donations au clergé. Ils nous font pénétrer dans l'intimité des familles : tel écuyer des écuries impériales lègue à sa femme et à son fils ses icônes et ses chevaux, après avoir coché, dans les listes, ce qui, des images pieuses et des montures, revient à chacun. À partir des dons faits aux églises par le défunt pour le salut de son âme, mais aussi d'indications fortuites, sur la situation de sa maison par exemple, il devient possible de repérer les relations de voisinage au sein d'une même paroisse, qui se confondent assez souvent avec des relations professionnelles: les hommes de cheval, par exemple, révèrent les saints jumelés Côme et Damien.

9 Les noms des témoins et des exécuteurs testamentaires, nécessairement proches du testateur, donnent des indications sur les réseaux d'amitié et d'alliance entre des personnages qui semblent se fréquenter sans tenir compte des barrières artificielles érigées par la hiérarchie moscovite. De nombreux testaments ont déjà été analysés et publiés, il est vrai, sans avoir recours aux registres de transactions patrimoniales. Mais il s'agissait le plus souvent de lignages nobles, surtout des mieux connus d'entre eux. Dans les registres apparaissent, pour la première fois de façon substantielle, des personnages de second plan comme des membres de la domesticité du palais, des centeniers de mousquetaires, des chargés d'affaires de monastères (monastyrskij strjapčij), des " chasseurs » du Service de la poste (jamskoj ohotnik), sans oublier les fondés de pouvoir, souvent de condition servile, qui représentent leur maitre lors de la transaction. À travers leur étude, on se prend à imaginer que sera comblé un jour le fossé qui, dans nos connaissances, sépare la noblesse des classes populaires, en restituant la physionomie de ce qu'on pourrait, avec beaucoup de précautions, qualifier de « classes moyennes ».

Les registres de transactions patrimoniales sont-ils utilisables pour une étude du marché de la terre? On peut en douter, pour plusieurs raisons. Les 2169 transactions décrites dans l'ouvrage ne représentent probablement qu'une fraction du marché foncier, donc un échantillon d'une réalité plus vaste, dont les dimensions mêmes nous échappent. La taxe de $3 \%$ ad valorem, dont devait s'acquitter le nouveau possesseur du bien au terme de la transaction, a probablement dissuadé la partie la moins fortunée des acheteurs d'avoir recours à l'enregistrement, et cela bien que la législation moscovite du XVII ${ }^{e}$ siècle exige de plus en plus, en cas de litige, la preuve du document écrit. Pour payer moins de droits, les acheteurs pouvaient aussi être tentés de déclarer des prix d'achat inférieurs à la réalité ; il est vrai qu'ils rendaient alors plus facile le « retrait lignager ", c'est-à-dire le rachat, par les parents proches du vendeur, du bien à son prix de vente dans un délai de quarante ans. Il est difficile enfin de calculer un prix moyen de la terre, à plus forte raison de suivre son évolution, tant le prix de vente dépend d'une multitude de facteurs: la qualité des sols, la nature et la valeur des bâtiments, la présence et le nombre de feux 
paysans. Autant d'obstacles qui se dressent sur le chemin de l'historien de l'économie, mais dont rien ne nous dit qu'ils soient insurmontables.

11 L'inventaire des registres de transactions patrimoniales arrive à son heure : depuis qu'elle existe, l'histoire de Russie a privilégié comme sujets d'étude, simultanément ou successivement, l'État, les grands mouvements de l'économie ou de la société, les guerres, la lutte des classes, l'évolution de la culture. Depuis quelque temps (vingt ans? trente ans?), l'attention des spécialistes commence à se tourner vers l'histoire privée. Nul doute que cette nouvelle publication les y aidera puissamment.

\section{NOTES}

1. RGADA : Rossijskij gosudarstvennyj arhiv drevnih aktov [Archives d'État des actes anciens de Russie], Moscou. Anton Antonov a déjà publié un recueil de documents extraits des registres et des rouleaux du Pomestnyj prikaz concernant les familles nobles (Akty služilyh zemlevladel'cev XV-načala XVII veka, vol. I-IV, 1997-2008, Arheografičeskij Centr, puis Drevnehranilišče) et les cadastres de Tver' ${ }^{\prime}$ au $\mathrm{XvI}^{\mathrm{e}}$ siècle (Piscovye materialy Tverskogo uezda XVI veka, Moscou, Drevnehranilišče, 2005). Il dirige une publication à périodicité irrégulière, Russkij diplomatarij (10 vol. parus, Arheografičeskij Centr, puis Drevnehranilišče, Moscou, 1997-2004), à laquelle il collabore abondamment. 